\title{
Do Varieties of Spanish Influence U.S. Spanish-English Bilingual Children's Friendship Judgments?
}

\author{
Maria M. Arredondo and Susan A. Gelman \\ University of Michigan
}

\begin{abstract}
Latinos are the largest minority group in the United States (U.S. Census, 2014), yet this term comprises individuals from multiple ethnicities who speak distinct varieties of Spanish. We investigated whether SpanishEnglish bilingual children $(N=140$, ages $4-17)$ use Spanish varieties in their social judgments. The findings revealed that children distinguished varieties of Spanish but did not use Spanish dialects to make third-person friendship judgments until 10-12 years; this effect became stronger in adolescence. In contrast, young children (4-6 years) made friendship judgments based on a speaker's language (English, Spanish). Thus, using language varieties as a social category and as a basis for making social inferences is a complex result of multiple influences for Spanish-speaking children growing up bilingual in the United States.
\end{abstract}

Starting in infancy, humans use social categories (e.g., gender and race) to organize information about people (Diesendruck \& haLevi, 2006; Kinzler, Shutts, \& Correll, 2010; Roberts \& Gelman, 2015; Taylor, Rhodes, \& Gelman, 2009; Waxman, 2012). Social categories allow children not only to identify those who share physical, behavioral, and psychological traits (Gil-White, 2001; Hirschfeld, 1996; Taylor, 1996; Taylor et al., 2009) but also to mark interpersonal obligations (Rhodes, 2012, 2013). Thus, social categories contribute to human behavior by shaping social relationships and their interactions, including friendship preferences, competition, and helpful or harmful behavior (Rhodes \& Chalik, 2013; Roberts, Williams, \& Gelman, 2016).

Among the attributes that contribute to social categorizations, language is a powerful cue. An exemplar-based model of speech perception suggests that traces of language (e.g., phonemes and lexical items) are implicitly stored along with information about a speaker and the social context to

The authors thank the University of Michigan Department of Psychology and Rackham Graduate School, the En Nuestra Lengua Literacy and Culture Program in Ann Arbor, Bennett Elementary School in Southwest Detroit, participating children and their families. We also thank the Conceptual Development Lab, especially Mélanie Rosado, Carolina Fuentes, Akemi Tsutsumi, Juna Kim, Di Xie, and Guadalupe Avila, for their assistance with data collection. Maria M. Arredondo thanks the National Science Foundation (NSF) Graduate Research Fellowship (grant no. DGE 1256260); any opinions, conclusions, or recommendations expressed in this material are those of the author(s) and do not necessarily reflect the views of NSF.

Correspondence concerning this article should be addressed to Maria M. Arredondo, 530 Church St., 2036 East Hall, Ann Arbor, MI 48109-1043. Electronic mail may be sent to mmarre@ umich.edu. help us identify individuals and social groups (Johnson, 2006). Language and accent can provide rich information about a speaker's origins, ethnicity, and even social status (Clopper \& Pisoni, 2004a; Labov, 2006; Van Bezooijen \& Gooskens, 1999). Children are able to distinguish language and accents as early as infancy and form inferences about others on the basis of these features (Kinzler, Dupoux, \& Spelke, 2007, 2012). By 3-5 years of age, children infer that those who share an accent live in the same geographic location but do not necessarily share personal preferences (e.g., activity or a game; Weatherhead, White, \& Friedman, 2016). By 45 years of age, children rely on accents to infer cultural norms (Weatherhead et al., 2016). Importantly, children also make social judgments based on language differences. For example, babies prefer to receive objects from native speakers over those who speak a foreign language or with a foreign accent (Girard, Floccia, \& Goslin, 2008; Kinzler et al., 2007; Kinzler, Dupoux, et al., 2012). In addition, when accent is pitted against race, children select as friends those who speak their accent (Kinzler, Shutts, DeJesus, \& Spelke, 2009; Kinzler et al., 2007).

Prior research examining children's use of language and accent to mark social groups has focused on monolingual children's first-person friendship preferences, in which a speaker of the child's own language is contrasted with a speaker of an

(C) 2017 The Authors

Child Development (C) 2017 Society for Research in Child Development, Inc. All rights reserved. 0009-3920/2019/9002-0023

DOI: $10.1111 /$ cdev.12932 
unknown language (e.g., English-speaking children were asked to consider speakers of English vs. French; Kinzler \& Dautel, 2012), or a native speaker of the child's own language is contrasted with a nonnative speaker of the same language (e.g., American English-speaking children were asked to consider a native speaker of English vs. someone speaking English with a French accent; Kinzler et al., 2007), or someone speaking in the child's local dialect is contrasted with someone speaking another dialect of that language (Kinzler \& DeJesus, 2013). This latter study revealed that when Englishspeaking children from Northern and Southern areas of the United States were asked to consider speakers with accents from those regions, only Northern 5- to 6-year-old children showed a preference for speakers of their own dialect and Southern children were at chance (Kinzler \& DeJesus, 2013). Thus, it is inconclusive whether children are sensitive to dialect varieties of their own language and whether they apply social judgments to such dialect variation early in development. A further limitation of past work is that dialect variation of English speakers within the United States, though meaningful, are regional and not broadly national, whereas Spanish speakers in the United States have experiences with dialects of Spanish from different countries. Thus, an important issue that has also begun to receive attention is how bilingual children use language and accent to guide their social judgments.

Spanish-English bilingual children in the United States interact daily with individuals who speak one or both of their languages in various dialects. A number of studies have documented that young bilingual children are aware that their two languages are different and use them appropriately with monolingual speakers (Nicoladis, 1998; Petitto et al., 2001; Tare \& Gelman, 2010). Byers-Heinlein (2014) proposed that bilinguals treat elements of each of their languages (e.g., sounds, words, and utterances) as belonging to separate categories. Furthermore, bilinguals link both of their languages to meaningful social categories, in their social judgments and preferences. For example, as with monolingual children (Kinzler et al., 2007, 2009), bilinguals prefer to receive toys from or befriend native-accented speakers rather than speakers of an unfamiliar language or accent (Cohen \& Haun, 2013; Kinzler, Shutts, \& Spelke, 2012; Souza, ByersHeinlein, \& Poulin-Dubois, 2013). However, if the two languages a child speaks are pitted against one another, then bilinguals show a preference for speakers of their dominant (most proficient) language (Byers-Heinlein, Behrend, Said, Giris, \& Poulin-Dubois, 2016) or speakers of the language that carries more prestige (Kinzler, Shutts, et al., 2012).

These findings demonstrate an early-emerging sensitivity to language differences as marking social relationships, in both monolingual and bilingual children. A central goal of this study is to examine bilingual children's awareness of their languages or dialects and how these ways of speaking are assigned to social relationships. In the United States, a child raised in a home where a non-English language is typically acquired first is on the path to being functionally bilingual (to some degree) in a heritage language and in English (Valdés, 2001). For bilinguals, proficiency in the majority language is traditionally treated as a pivotal measure of the degree to which an individual has acculturated, or assimilated, to the majority culture (Félix-Ortiz, Newcomb, \& Myers, 1994). In contrast, little is known about whether young bilingual children make use of dialect differences in their heritage language to infer social interactions among speakers. A particularly important arena for addressing these issues concern Spanish-English bilingual children in the United States.

\section{Spanish-Language Speakers in the United States}

Individuals of Spanish-speaking descent, also known as Hispanics or Latinos, are the largest minority group in the United States, numbering at about 54 million and $17 \%$ of the total population (U.S. Census, 2014). It is estimated that about 70\% of Latinos over the age of 5 speak Spanish at home (U.S. Census, 2014). The umbrella terms "Hispanics" or "Latinos" are often used interchangeably (although "Hispanic" was implemented for policy purposes, and "Latino" as a form of self-identification) and refer to individuals whose ethnic backgrounds may originate from multiple countries in Latin America and/or Spain (Oboler, 1995). Critics argue that the use of single "pan-ethnic" terms overlook the diverse experiences and cultures of specific populations, and neglect to contextualize their history in the United States (Gimenez, 1989). For example, Mexicans (64\%) and Puerto Ricans $(10 \%)$ are two of the largest Latino ethnic groups, yet their immigrant histories differ markedly. Whereas Puerto Rico is an unincorporated U.S. territory, the current U.S. southwest region was originally part of Mexico, which explains Mexicans' long-standing presence in the region (Gutiérrez, 2004). 
Despite the existence of pan-ethnic terms to unify multiple ethnicities, individuals belonging to these ethnic groups often do not strongly identify with these terms (Feliciano, 2009; Masuoka, 2006; Taylor, Lopez, Martínez, \& Velasco, 2012). Instead, Latinos have typically used their family's country of origin as a form of identification and report that their shared connection to other Latinos is often the use of Spanish (Geerlings, Verkuyten, \& Thijs, 2014; Taylor et al., 2012). Indeed, Spanish speakers often reveal their ethnicity via their dialects, including vocabulary, pronunciation, and grammatical features. For example, there are 23 ways of saying "bus" across Spanish-speaking countries (e.g., taxivan [Chile, Paraguay, Uruguay], colectivo or omnibus [Argentina], chiva [Colombia, Panamá, Perú], camión or micro [Mexico], bús [Costa Rica], and wawa [Puerto Rico, Cuba]). Also, whereas in Mexico the $/ r /$ is assibilated, in Puerto Rico its sound is a velarized trill and occasionally closer to the French uvular /r/ (Hualde, 2005; Resnick, 1980). As a grammatical example, the pronoun for second-person singular varies across Latin America in the use of vos (predominant in Argentina, Uruguay, eastern Bolivia, Paraguay, El Salvador), tú (Mexico, Perú), and usted (Venezuela, Colombia, Costa Rica).

Given the landscape of Spanish-language differences, an important unanswered question concerns when in development Spanish-speaking children who are living in the United States differentiate varieties of Spanish to mark social categories and use them to make social judgments. In the remainder of the article, we do not use the terms Hispanic or Latino, instead we use "Spanish-English bilinguals," which we believe describes a similar sociolinguistic experience for those living in the United States and are part of this group, although we understand that this terminology does not suffice to describe their cultural experiences.

\section{The Present Studies}

The present studies investigated whether and when two different varieties of Spanish affect the social judgments of Spanish-English bilingual children living in the United States. To examine this question, we employed a task developed by Kinzler et al. $(2007,2009)$ that was developed to investigate first-person social preferences (whom participants would prefer to play with), whereas the present studies elicited third-person social judgments (whom another child would prefer to play with). In prior work, when race and language were pitted against one another, children typically reported that they wanted to be friends with those who spoke the same language or with the same accent, regardless of race (Kinzler et al., 2007, 2009). Pitting conflicting social cues against one another in a third-person task allowed us to examine the importance of language variety against both race and gender, in a fully counterbalanced design, in order to determine the relative value of different social cues for bilingual children when making friendship judgments.

By adulthood, Spanish-speaking individuals distinguish national ethnic backgrounds using a speaker's language dialect. Thus, we predicted that at some point in development, Spanish-English bilingual children would be aware of distinct Spanish varieties and use them to determine friendship judgments. Given that prior work shows that language varieties are used to make social inferences as early as infancy, it is possible that SpanishEnglish bilingual children would be sensitive to Spanish dialects early in development. However, given that Spanish is a minority language in the United States, it is also possible that bilingual children would treat any dialect differences in Spanish as minimal when making social inferences, and thus any sensitivity could increase with age.

We selected Mexican and Puerto Rican dialects as the two varieties of Spanish, because Mexicans and Puerto Ricans are the two largest Latino ethnic groups in the United States, and their dialects differ from one another phonologically. For example, in Mexico, there is a strong sibilant pronunciation of syllable and word final /s/, whereas in Puerto Rico, $/ s /$ is weakened to an aspiration $[h]$ or deleted; in Mexico the word-final $/ n /$ is alveolar, whereas in Puerto Rico, it is velarized; in Mexico, $/ r /$ is an alveolar trill, whereas in Puerto Rico, $/ r /$ is a velarized trill and occasionally closer to the French uvular $/ r /$; also in Mexico, intervocalic $/ j /$ is weak and may disappear (e.g., gallina, sello), whereas in Puerto Rico, it is given an affricate pronunciation initially and does not weaken intervocally (Resnick, 1980).

\section{Study 1: Bilingual Children's Use of Spanish Dialects During Friendship Judgments}

Building on prior research showing that bilingual and monolingual children are sensitive to accented speakers when making first-person social judgments in the majority language (Kinzler, Dupoux, et al., 2012; Kinzler, Shutts, et al., 2012; Souza et al., 2013), Study 1 examined whether Spanish-English 
bilingual children are sensitive to the varieties of dialects in their heritage language (i.e., Spanish) and whether they use it to make third-person friendship inferences. Spanish-English bilingual children, ranging from 4 to 17 years, were asked to make friendship judgments on the basis of differing Spanish dialects (Mexican and Puerto Rican Spanish) when dialect was crossed with the social categories of gender and race.

\section{Method}

\section{Participants}

Seventy-seven Spanish-English bilingual participants took part in the study. Participants were from four age groups: 4-6 years $(N=20,12$ girls, $\left.M_{\text {age }}=5.7, S D=0.65\right), 7-9$ years $(N=22,14$ girls, $M_{\text {age }}=8.6$ years, $\left.S D=0.67\right), 10-12$ years $(N=20$, 11 girls, $M_{\text {age }}=11.1$ years, $\left.S D=0.78\right)$, and $13-$ 17 years $\left(N=15,10\right.$ girls, $\left.M_{\text {age }}=15.1, S D=1.2\right)$. Two 4- to 6-year-old children were excluded from the final sample as they did not pass the Spanish proficiency assessment during the initial portion of the testing session, thus testing stopped for these children and they did not complete the remaining tasks (see Method in the following section). In addition, four participants (one in each age group) were excluded from the final sample because their choices during the Friendship Judgment task were one-sided (left throughout or right throughout) from the two choices presented.

Children's families in the final sample identified one or both parents as Hispanic: $47.26 \%$ Mexican, $18.49 \%$ from South America, 17.8\% from Central America or the Caribbean (including Puerto Rico), $4.11 \%$ from Spain, $6.85 \%$ from the United States, and $5.48 \%$ from another non-Spanish-speaking country. Child participants received daily exposure to both English and Spanish, and heard Spanish from at least one parent who was a native Spanish speaker and reported consistent use of Spanish at home with their child(ren). Data collection took place between August 2013 and January 2015. All families were recruited from the same neighborhoods in a suburban town located in southeast Michigan and were of similar socioeconomic status (Table 1). In their language of choice (English or Spanish), parents provided consent, children under the age of 13 provided verbal assent, and adolescents provided written assent. As a token of our appreciation for their participation, families received monetary compensation and children received a small toy.

\section{Materials}

Parent questionnaires. Parents completed a questionnaire about their child's bilingual language development, the mother's educational level, household income, and two subjective measures of social status in their community and in the United States (from the John D. and Catherine T. MacArthur Foundation Research Network on Socioeconomic Status and Health questionnaire, retrieved from: www.macses.ucsf.edu). Parents also completed three scales assessing essentialist beliefs regarding race, gender, and ethnicity (Rhodes \& Gelman, 2009) for exploratory purposes only and so these results are not reported. All questionnaires were available in English and Spanish for parents to complete in the language of their choice. All questionnaires were originally developed in English and translated to Spanish by the first author, then a native Spanishspeaking research assistant back translated all forms to English; any mistranslations were discussed to choose better wording for Spanish forms.

Experimental child session. Images from Richard Scarry's (2011) Best Busy Year Ever and an Olympus VN-7200 (Olympus Cooperation of the Americas, Center Valley, PA, USA) digital voice recorder were used for assessing children's Spanish proficiency. During the Friendship Judgment and Dialect Judgment tasks, children wore a Plantronics (Plantronics, Inc, Santa Cruz, CA, USA) Audio 355 headset to clearly hear sound clips.

The Friendship Judgment task was presented in Microsoft PowerPoint on a 13-in. MacBook laptop (Apple Inc, Cupertino, CA, USA). Trials consisted of voice clips recorded in Spanish by children living in either Mexico (two girls and two boys) or Puerto Rico (two girls and two boys). The content of all sound clips was intended to be neutral and not revealing of personal preferences (e.g., La gente camina por la calle; English translation: People walk down the street). The Mexican versus Puerto Rican clips differed from one another in intonation and pronunciation (see Introduction for the key phonological differences). Sound clips of Spanish dialects were pretested with 20 native Spanish-speaking adults (10 female; $M_{\text {age }}=26.3$ years; $S D=6.5$ ), who judged where the speakers were from. Sound clips with accuracy above $85 \%$ were selected as part of the sound stimuli in the task (overall accuracy for selected sound clips was 92.5\%). See Appendix S1 for stimuli list.

Images of Black and White boys and girls were selected from previous research (Kinzler et al., 2009) and Corbis Images. Sixteen triads were 
created so that each target matched one test image in linguistic variety (Mexican or Puerto Rican Spanish) but not race (eight trials, race mismatch condition) or gender (eight trials, gender mismatch condition), and the other test image matched in race or gender but not linguistic variety; see Appendix S1 for stimuli list. Gender mismatch triads consisted of a target (either a boy or a girl) along with one male and one female test image; within a triad, all three were the same race. Race mismatch triads consisted of a target (either a Black or White child) along with one Black and one White test image; within a triad, all three were the same gender. The three factors (gender, race, and dialect) were fully crossed, and each combination was represented twice for both race and gender mismatch triads. During each triad, the target image appeared in the center of the top half of the computer screen and one testing item in the bottom left and the other testing item in the bottom right of the screen (Figure 1). Order of triads was randomized and the lateral positions of languagematching and gender- or race-matching test images were orthogonally counterbalanced. On any given trial, the three sound clips that participants heard were of different content.

For each trial, children were given a score of 1 each time they selected a testing image that matched the Target's Spanish dialect (Mexican or Puerto Rican Spanish), and a score of 0 each time they selected the other image that matched race during the race mismatch condition or gender during the gender mismatch condition.

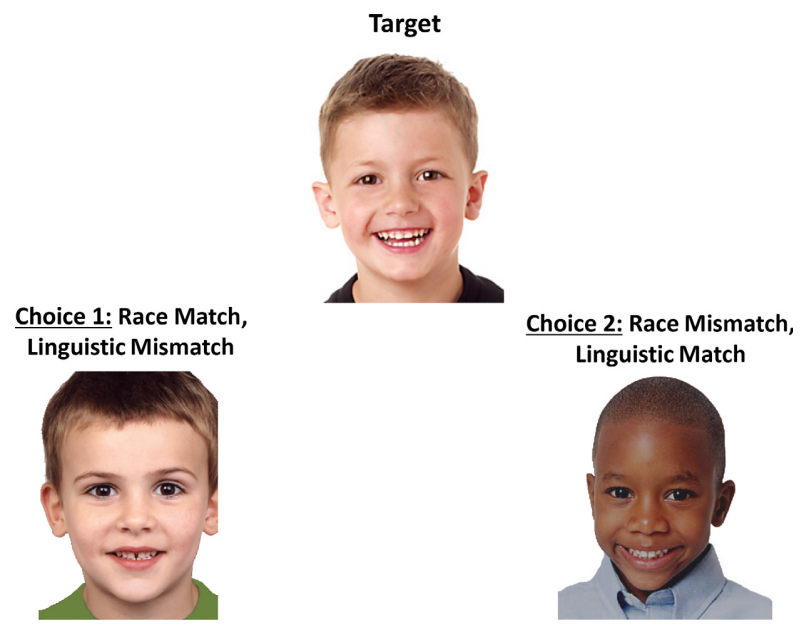

Figure 1. Example trial for race mismatch condition. Choice 1 matches the target's race but does not match the target's linguistic variety. Choice 2 does not match the target's race but matches the target's linguistic variety. [Color figure can be viewed at wileyonlinelibrary.com]
A Dialect Judgment task assessed whether children could identify the speaker's dialect, for each of 16 randomized Spanish sound clips used in the Friendship Judgment task (eight Mexican Spanish and eight Puerto Rican Spanish trials). Children completed this task after the Friendship Judgment task so as not to bias children to make friendship inferences based on geographical location. For each trial, children were given a score of 1 each time they named the correct country of origin for sound clips and a score of 0 each time they selected the incorrect country.

\section{Procedure}

The sessions were carried out entirely in Spanish, with a native Spanish-speaking experimenter. At the beginning of the session, children were presented with images from Richard Scarry's Best Busy Year Ever and were asked to tell the experimenter what they saw in the images for $\sim 90 \mathrm{~s}$. This task was designed to assess children's Spanish proficiency; children who did not respond in Spanish sentences of at least three words in length did not proceed to the remaining tasks. Responses were audio recorded for later coding to confirm that the child spoke Spanish.

Before testing for the Friendship Judgment task began, the experimenter explained to the child that they would be seeing some children, hear them speak, and pick which ones would play together. During each triad, the experimenter pointed to the target image and stated that the child would be picking someone to play with the Target and that they would hear what they sound like (Vamos a escoger a alguien para que juegue con este niño. Este niño habla así. Translation: "Let's pick a playmate for this child, who sounds like this."). Next, the experimenter pointed to the bottom left testing image and asked the child to hear what that one sounds like, followed by the bottom right testing image (Este habla así. Translation: "This one sounds like this"). Then, the experimenter pointed to both testing images twice while asking the child which of the two the Target would like to play with $(i C o n$ cuál va a querer jugar? Translation: "Which one would this one like to play with?"). Children indicated their choice by pointing to one of the testing images. Before testing, children were also presented with two practice triads of cats and dogs along with respective audio clips (meows and barks) and similar testing instructions.

Following the Friendship Judgment task, all children completed the Dialect Judgment task. During this task, the experimenter explained that there are 
two different places named "Mexico" and "Puerto Rico" that are far from each other, that both have people that speak Spanish, but that it is spoken a bit differently in the two places. Then, the experimenter allowed the children to hear two sound clips, one of a child from Mexico and another of a child from Puerto Rico (labeled as such), which were not included during any portion of the Dialect Judgment task testing session. Children were then presented with 16 sound clips from the "Friendship Judgment task" and were asked to name where the speaking children were from. Every four trials children were allowed to hear the same two sound clips that were initially heard, as a reminder of what children from Mexico and Puerto Rico sound like.

\section{Results}

See Figure 2 for means, standard errors, and significance results from nonparametric one-sample Wilcoxon signed-rank tests (two-tailed, $p<.05$ ) examining performance against chance (.50) for each mismatch condition (race and gender). We conducted a generalized estimating equation (GEE) analysis, specifically a repeated measures generalized logistic regression, using the mismatch conditions and age groups as factors. The analysis revealed a significant main effect of age group (Wald $\chi^{2}=22.60, d f=3, p<.001$ ) but not condition (Wald $\chi^{2}=0.10, d f=1, p=.75$ ). Post hoc analyses using a Bonferroni correction revealed that 13- to

\section{A}

\section{Gender Mismatch}

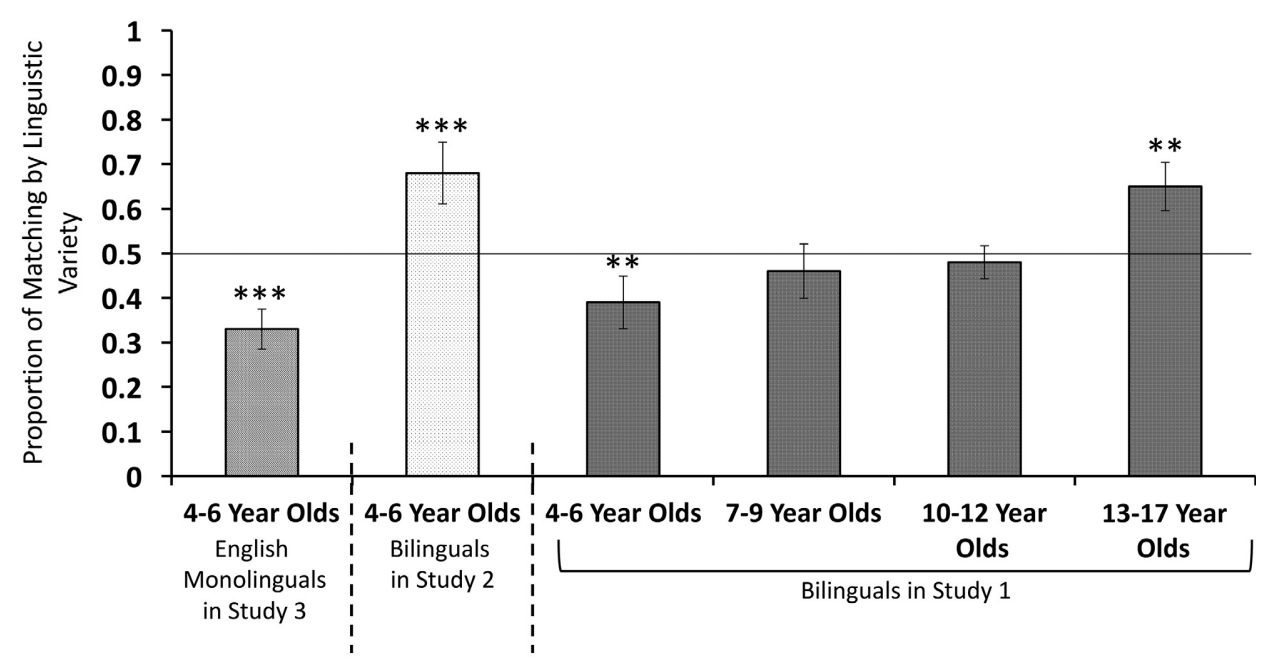

B

Race Mismatch

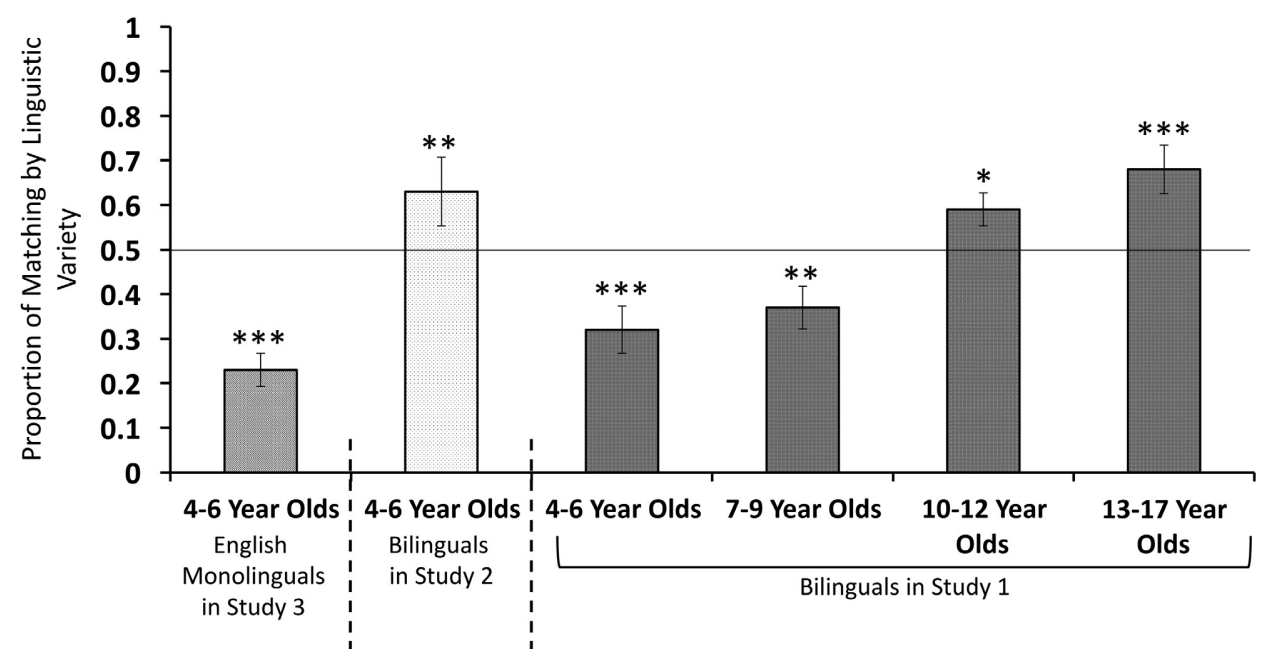

Figure 2. Results from (A) gender mismatch and (B) race mismatch conditions. Bars depict standard error. ${ }^{*} p<.05,{ }^{* *} p<.01$, $*_{* * *}<.001$ indicates the result of a Wilcoxon signed-rank one-sample $t$-test to chance $(0.50)$. 
17-year-olds $(M=0.66, S D=0.47)$ made selections based on dialect significantly more than 4- to 6-yearolds $(M=0.35, S D=0.48, p<.001)$ and 7- to 9-yearolds $(M=0.41, S D=0.49, p=.001)$. Also, 10 - to 12-year-olds $(M=0.54, S D=0.50)$ made selections based on dialect significantly more than 4 - to 6-yearolds $(p=.01)$; remaining post hoc comparisons were not significant. The analyses also revealed a significant Age Group $\times$ Mismatch Condition interaction, Wald $\chi^{2}=11.83, d f=3, p=.008$. Post hoc analyses using a Bonferroni correction revealed that the interaction stemmed from developmental differences when matching dialects based on the conditions; specifically the two oldest age groups (10-12 and 1317) were significantly more likely to make selections based on dialect during the race mismatch condition, in comparison to younger children (4-6: $p=.001$ and $p<.001 ; 7-9: p=.005$ and $p<.001$, respectively). Yet this was not the case during the gender mismatch condition, as only 13- to 17-year-olds were significantly more likely to make selections based on dialect, in comparison with 4- to 6-year-olds: $p=.028$. The remaining post hoc comparisons were not significant.

One-sample Wilcoxon signed-rank tests revealed that children in all age groups were able to differentiate the country of origin for sound clips during the Dialect Judgment task; see Figure 3 for significance results on two-tailed tests $(p<.05)$ compared against chance (.50). A GEE generalized logistic regression using the age groups as factors revealed that performance was predicted by age group (Wald $\chi^{2}=52.76, d f=3, p<.001$ ), indicating steady improvement over the ages tested. Post hoc analyses using a Bonferroni correction revealed that the two oldest age groups (10-12 and 13-17) were significantly more likely to match voice sound clips to their correct country of origin than were younger children (4-6: $p \mathrm{~s}<.001 ; 7-9: \mathrm{ps} \leq .005)$. The youngest age groups (4-6 and 7-9) and the oldest age groups (10-12 and 13-17) did not significantly differ from one another in their accuracy; remaining post hoc comparisons were not significant.

A correlation between the Friendship and Dialect Judgment tasks revealed that children's performance on the two tasks was positively related, $r(73)=.30$, $p=.01$; however, after controlling for age, the partial correlation was nonsignificant. In addition, because roughly half the participants had at least one parent of Mexican background, we performed a post hoc analysis comparing those participants to those who did not have any Mexican background. As shown in Appendix S2, there were no significant differences on the Friendship Judgment task between the two groups, at any age.

One-way analyses of variance did not reveal differences across age groups for parents' reporting of their household income and subjective scales of social status; see Table 1 . However, a one-way analysis of variance revealed that mothers' education differed across the age groups, $F(3,63)=2.95$, $p=.039, \eta_{p}^{2}=.12$. Post hoc analyses using a Bonferroni correction revealed that mothers in the 4- to 6-year-old group were on average one level higher in education than those in the 7- to 9-year-old group $(p=.04)$; there were no other differences.

\section{Accent Judgment Task Performance}

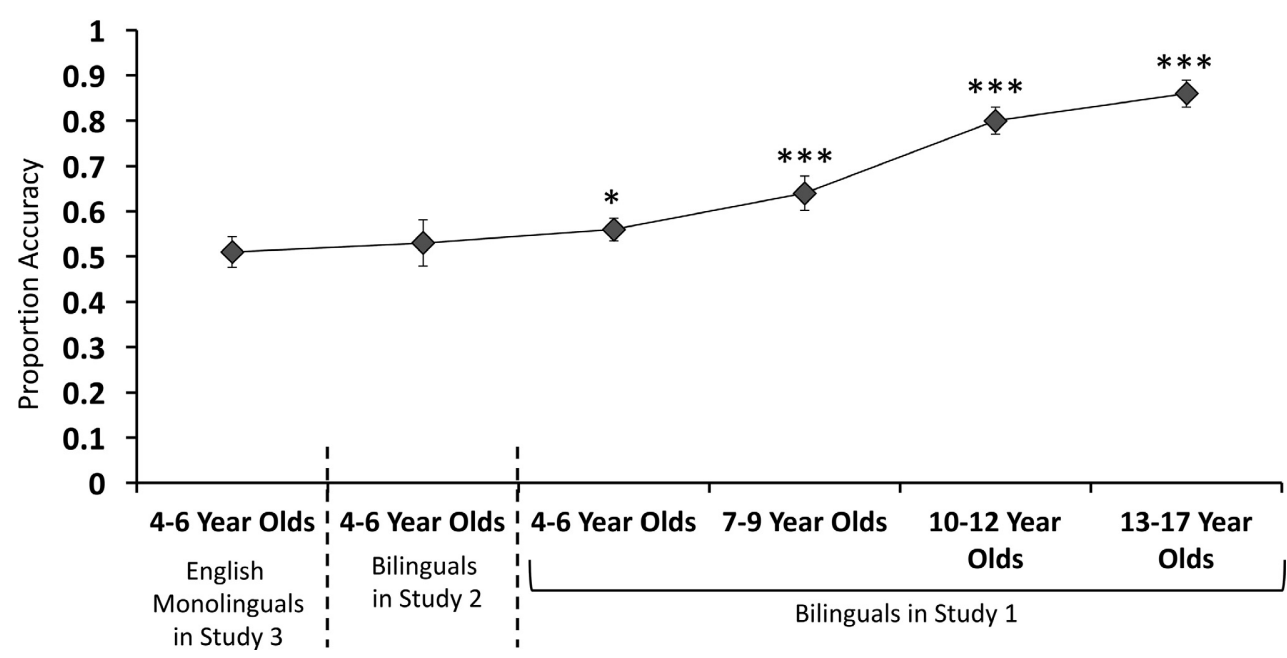

Figure 3. Average performance (standard errors in brackets) for the dialect judgment task among children who took part in Study 1 (4to 6-year, 7- to 9-year, 10- to 12-year, and 13- to 17-year olds), 4- to 6-year-old bilinguals in Study 2, and 4- to 6-year-old monolinguals in Study 3. ${ }^{*} p<.05$ and ${ }^{* *} p<.001$ indicate the result of a Wilcoxon signed-rank one-sample $t$-test against chance $(0.50)$. 


\section{Discussion}

Study 1 investigated whether and at what age Spanish-English bilingual children used varieties of Spanish to guide third-person friendship judgments. Results revealed that as bilingual children get older, distinct varieties of Spanish are increasingly used to determine friendship judgments when pitted against gender and racial social categories. Beginning at 10-12 years, children differentiated the country of origin for the sound clips in the Dialect Judgment task and predicted that children would be more likely to befriend those who spoke the same variety of Spanish. This pattern emerged earlier when language variety was pitted against race than when it was pitted against gender, suggesting that children are weighing the relative importance of language variety against other social identities rather than simply attending to language as a salient cue. Importantly, these findings indicate that, by adolescence, Spanish-English bilingual children living in the United States do not consider Spanish speakers (Hispanics or Latinos) as an undifferentiated social group but predict social consequences of different varieties in the Spanish language.

We obtained a positive relation between the Friendship and Dialect Judgment tasks, suggesting that, as children get better at distinguishing the Spanish dialects, they increasingly use them to make friendship inferences. The younger children in this study (4-6 and 7-9 years of age) did not predict friendship patterns on the basis of Spanish varieties, instead selecting primarily based on the social categories of gender or race. In part, this may reflect their difficulty attending to the different dialects that were presented: These younger children were much less successful at identifying which speech clips were "Mexican" or "Puerto Rican" on the Dialect Judgment task, as compared with the older age groups. Nonetheless, even the youngest children were above chance on the Dialect Judgment task, suggesting that even when children could detect the difference, they did not use it to guide their social judgments. It is, thus, important to determine whether this third-person social judgment task is within the capacity of these young bilingual children. This was the goal of Study 2, to which we turn next.

\section{Study 2: Bilingual Children's Use of Spanish Versus English Languages}

Study 2 was designed as a companion to Study 1, to test whether children 4-6 years (the youngest age group in that study) attend to language during social judgments, when the language contrast is between two languages (Spanish vs. English) rather than two dialects (Mexican Spanish vs. Puerto Rican Spanish). As in Study 1, we tested SpanishEnglish bilingual children's third-person friendship inferences when language was pitted against a social category based on race or gender. The only difference from Study 1 was that instead of contrasting dialects, we contrasted languages. If young children use the distinction between Spanish and English to guide their predictions about friendship patterns, then this would suggest that children's performance in Study 1 was likely due to the contrast between different varieties of Spanish. In contrast, if children do not use the distinction between Spanish and English to guide their friendship judgments, then this would suggest that their difficulty in Study 1 was not due to reasoning about varieties of Spanish per se. In such a case, performance could be due either to participants' bilingual environments in which both languages are learned simultaneously or the higher cognitive demands of the third-person task.

\section{Method}

\section{Participants}

A new group of 20 Spanish-English bilingual children ages 4-6 years (10 girls; $M_{\text {age }}=5.7$ years; $S D=0.98$ ) took part in this study, all raised and educated in the same suburban town located in southeast Michigan as children in Study 1. One child was excluded from the final sample, as they did not pass the Spanish proficiency assessment during the initial portion of the testing session. At the time of testing, children were receiving daily exposure to both languages (Spanish in the home and English outside the home). At least one parent was a native Spanish speaker and reported consistent use of Spanish at home with their child(ren). Children's families identified one or both parents as Hispanic: $12.5 \%$ Mexican, 37.5\% from South America, $18.75 \%$ from Central America or the Caribbean (including Puerto Rico), 9.38\% from Spain, 18.75\% from the United States, and 3.12\% from a nonSpanish-speaking country. Data collection took place between March 2014 and January 2015. Parents provided consent and children provided assent in their language of choice, English or Spanish. As a token of our appreciation for their participation, families received monetary compensation and children received a small toy. 


\section{Materials and Procedure}

The materials and procedure were mostly identical to those of Study 1, except that the Friendship Judgment task contrasted English versus Spanish rather than two varieties of Spanish. Four SpanishEnglish bilingual children (two boys and two girls) recorded sound clips in English for use in this task. The Dialect Judgment task was identical to that of Study 1 and included the Spanish dialect sound clips.

\section{Results}

We conducted a GEE repeated measures generalized logistic regression using the mismatch conditions as a factor, which revealed that children did not differ in performance between the gender and race mismatch condition (Wald $\chi^{2}=2.03, d f=1$, $p=.154)$; Figure 2 .

Of particular interest was how performance in Study 2 compared with that of the same age group of children from Study 1; thus, we carried out a GEE repeated-measures generalized logistic regression using the mismatch conditions (race, gender) and Study (1 [Dialect], 2 [Language]) as factors. The analysis revealed that study predicted performance (Wald $\chi^{2}=10.76, d f=1, p=.001$ ); post hoc analyses using a Bonferroni correction revealed that children in this study (Language version, $M=0.65$, $S D=0.48)$ matched by the target's language more than those in Study 1 matched by dialect $(M=0.35$, $S D=0.48, p<.001)$. The analysis also revealed that mismatch condition predicted performance (Wald $\chi^{2}=4.08, d f=1, p=.043$ ); post hoc analyses using a Bonferroni correction revealed that children were more likely to match by language/dialect during the gender mismatch condition $(M=0.54$, $S D=0.50)$ than the race mismatch condition $(M=0.47, S D=0.50, p=.043)$. The analysis did not reveal a significant Study $\times$ Mismatch Condition interaction, Wald $\chi^{2}=0.06, d f=1, p=.81$.

Analyses on the Dialect Judgment task revealed that children in this study performed at chance levels (Figure 3). A GEE generalized logistic regression using Study (1 [Dialect], 2 [Language]) as a between-subjects factor did not reveal differences in performance by Study (Wald $\chi^{2}=0.54, d f=1$, $p=.463)$, indicating that children in the two groups performed similarly.

Independent-samples $t$-tests did not reveal differences in parents' reporting of household income and subjective scales of social status from those of Study 1 (Table 1). However, mothers' education in this study was significantly higher than that in Study $1, t(34)=2.28, p=.029$.

\section{Discussion}

Study 2 examined whether Spanish-English bilingual preschoolers living in the United States made third-person friendship inferences based on language (English vs. Spanish). Children were significantly more likely to match the Target image to an option based on language in Study 2 than by Spanish dialect in Study 1. Thus, 4- to 6-year-olds are capable of making third-person judgments, and their performance in Study 1 is not due to difficulty in making third-person inferences. Instead, 4- to 6year-old children detect dialect differences in their heritage language (as seen in Study 1) but place greater weight on language differences. This may be because children do not detect dialect differences as readily as language differences in early childhood, and/or because they detect them but do not give them as much weight when making third-person inferences.

\section{Study 3: English Monolingual Children's Use of Spanish Dialects}

In Study 1, a sample of young Spanish-English bilingual children in the United States were not likely to use Spanish dialects to guide their social judgments. However, their performance was not at zero, thus raising the question of whether or not they made use of dialect more than would a group with no prior exposure to Spanish. Thus, in order to better interpret the performance of the youngest age group of children in Study 1, we tested a group of monolingual English-speaking children of the same age, as a baseline comparison group. The relative performance of bilingual and monolingual English-speaking children enabled us to assess whether the bilingual children were making any use of Spanish variety above a sample of children who had not been exposed to Spanish.

\section{Method}

\section{Participants}

Thirty-seven English monolingual children ages 4-6 years (21 girls; $M_{\text {age }}=5.68$ years; $S D=0.67$ ) took part in the study, all raised and educated in a suburban town located in southeast Michigan; families were recruited from the same neighborhoods as 
bilinguals in Studies 1 and 2. Only a subset of the monolingual children participated in the Dialect Judgment task ( $n=17$ of the larger sample of 37 ); we informally noticed that these children seemed to find the Dialect Judgment task confusing, often saying "I don't know," asking when the task would end, looking anxious, and/or apparently guessing by alternating responses. Data collection took place between June-July 2015 and August 2016. At the time of testing, all parents reported that children had never been exposed to another language except English. Most parents identified as White (83.8\%), $1.35 \%$ as Black, $2.7 \%$ as Asian, $9.45 \%$ as mixed race, and $2.7 \%$ chose not to answer. As a token of our appreciation for their participation, families received monetary compensation and children received a small toy.

\section{Materials and Procedure}

The experimenter spoke to children and their families exclusively in English, while the stimuli presented were in Spanish. Children completed the book session using Richard Scarry's (1991) Best Busy Year Ever in English and the Spanish Dialects version for the Friendship Judgment task; materials and procedure were identical to those of Study 1.

\section{Results}

We conducted a GEE repeated-measures generalized logistic regression using the mismatch condition as a factor. The analysis revealed a mismatch condition effect (Wald $\chi^{2}=8.83, d f=1$, $p=.003)$, in which children were more likely to make selections based on dialect during the gender than race mismatch condition. See Figure 2 for results.

Of particular interest was how performance for English monolingual children compared with that of the same age group of bilingual children from Study 1 ; thus, we carried out a GEE repeated-measures generalized logistic regression using the mismatch conditions (race, gender) and study (1 [bilingual], 3 [monolingual]) as factors. This analysis revealed an effect of mismatch condition (Wald $\chi^{2}=9.103$, $d f=1, p=.003)$, in which children were more likely to make selections based on dialect during the gender $(M=0.36, S D=0.48)$ than race mismatch condition $(M=0.27, S D=0.43)$. We did not find a main effect of study; that is, there were no differences in performance between monolinguals and bilinguals, nor a Study $\times$ Mismatch interaction.

Analyses on the Dialect Judgment task revealed that children in this study performed at chance levels; see Figure 3. A GEE generalized logistic regression using Study (1 [bilinguals], 3 [monolinguals]) as a between-subjects factor did not reveal differences by Study in the Dialect Judgment task performance (Wald $\chi^{2}=1.69, d f=1, p=.193$ ), indicating that children in the two groups performed similarly. In addition, independent-samples $t$-tests between monolinguals' and bilinguals' parents did not reveal differences in mothers' education and reporting of subjective scales of their community and national ladder; however, family income was significantly higher for monolingual families, $t(43)=4.14, p<.001$; see Table 1 .

Table 1

Averages (Standard Deviation; Sample Size [n]) for Parents' Demographic Responses for Studies 1-3

\begin{tabular}{|c|c|c|c|c|}
\hline & Income & Mother's education & Community scale & National scale \\
\hline \multicolumn{5}{|l|}{ Study 1} \\
\hline 4- to 6 -year olds $(n=20)$ & $6.33(2.09 ; 15)$ & $3.65(1.17 ; 17)$ & $7.56(1.47 ; 17)$ & $6.56(1.52 ; 17)$ \\
\hline 7- to 9-year olds $(n=22)$ & $5.85(2.06 ; 20)$ & $2.33(1.32 ; 21)$ & $7.57(2.03 ; 21)$ & $6.05(2.16 ; 21)$ \\
\hline 10 - to 12 -year olds $(n=20)$ & $6.37(1.96 ; 16)$ & $2.59(1.28 ; 17)$ & $8.00(1.46 ; 18)$ & $6.78(1.73 ; 18)$ \\
\hline 13 - to 17 -year olds $(n=15)$ & $6.91(2.29 ; 11)$ & $3.08(2.07 ; 12)$ & $7.00(1.71 ; 12)$ & $5.58(2.23 ; 12)$ \\
\hline \multicolumn{5}{|l|}{ Study 2} \\
\hline $\begin{array}{l}\text { 4- to 6-year olds }(n=20) \\
\text { Language condition }\end{array}$ & $7.12(1.83 ; 17)$ & $4.74(1.63 ; 19)$ & $7.95(2.56 ; 20)$ & $7.30(2.23 ; 20)$ \\
\hline \multicolumn{5}{|l|}{ Study 3} \\
\hline $\begin{array}{l}\text { English monolinguals } \\
\text { 4- to 6-year olds }(n=37)\end{array}$ & $8.23(1.01 ; 30)$ & $3.94(1.01 ; 36)$ & $7.23(1.61 ; 35)$ & $6.91(1.63 ; 35)$ \\
\hline
\end{tabular}

Note. Options for yearly household income were the following: (a) < \$5,000; (b) $\$ 5,000-\$ 11,999$; (c) $\$ 12,000-\$ 15,999$; (d) $\$ 16,000-\$ 24,999$; (e) $\$ 25,000-\$ 34,999$; (f) $\$ 35,000-\$ 49,999$; (g) 50,000-\$74,999; (h) $\$ 75,000-\$ 99,999$; (i) $\$ 100,000$ and greater. Options for mother's education were the following: (a) High school diploma or equivalent (GED), (b) Associate's degree, (c) Bachelor's degree, (d) Master's degree, (e) Doctorate degree (Ph.D), Professional degree (MD, DD, DDS, etc.). Community and National Scales were subjective measures of social status, in which parents placed themselves on an 11-step ladder, high numbers represent high status and low number represent low status. 


\section{Discussion}

Study 3 was designed to provide a baseline "control" sample to compare the performance of the youngest children (4-6 years) in Study 1. Specifically, we tested a sample of English monolingual children with no exposure to Spanish, to determine whether the bilingual Spanish-English speakers in Study 1 were any more likely to make third-person friendship inferences using linguistic varieties of Spanish. As expected, English monolingual preschoolers did not discriminate between the Spanish dialects nor did they use the Spanish dialects to guide their friendship judgments, and instead typically selected the member of the test pair that matched the racial or gender category of the target image. Of greatest interest, the youngest bilingual children from Study 1 were no more likely to discriminate the Spanish dialects or use them to make friendship judgments, than monolingual English speakers in Study 3.

\section{Study 4: Bilingual Children's Use of Spanish Dialects (Urban Sample)}

Children raised and educated in different cultural and social contexts can vary in their conceptualization of social categories (e.g., Diesendruck et al., 2013; Kinzler \& Dautel, 2012; Rhodes \& Gelman, 2009). An important consideration is whether being part of a community in which the child's heritage language and the ethnic composition are the majority may increase Spanish-English bilingual children's sensitivity to the various dialects in their heritage language. That is, children living in a social context that allows them greater exposure to a community of Spanish speakers outside the home may be more sensitive to its dialect variations and thus more likely to make social judgments on the basis of these linguistic cues. Children who took part in Studies 1-3 were from a suburban area in Southeast Michigan in which only $4 \%$ of the population in the area identified as Hispanic (U.S. Census, 2016). In Study 4, we included a sample of children living in a neighborhood called "Mexicantown" located in southwest Detroit that experienced a $70 \%$ increase of Hispanics in the prior 20 years (U.S. Census, 2014). The present study investigated whether children living in a community composed of multiple generations of Mexican immigrants, attending a school in which $68.4 \%$ were Spanish speakers, who have a greater likelihood of encountering individuals who speak one of the Spanish dialects tested in the study, differ in their sensitivity to Spanish dialects when making third-person friendship judgments. We also focused on 7- to 9- and 10- to 12-yearolds, as Study 1 found that 10- to 12-year-olds began using Spanish varieties to predict children's friendship judgments, whereas 7- to 9-year-olds did not.

\section{Method}

\section{Participants}

Forty-three children participated: 22 children ages 7-9 years (15 females; $M_{\text {age }}=8.5 ; S D=0.69$ ) and 21 children ages 10-12 years (15 females; $M_{\text {age }}=11.0 ; S D=0.49$ ). Four children (three 7 - to 9-year-olds, one 10- to 12-year-old) were excluded from the final sample, as they did not pass the Spanish proficiency assessment during the initial portion of the testing session. Children attended an elementary school in southwest Detroit in the area known as "Mexicantown" for its large Hispanic (but especially, Mexican) population that immigrated to the region beginning in the 1940s. At the time of data collection (April 2014-June 2015), the school's ethnic/racial distribution was $84.9 \%$ Hispanic or Latino, $8.3 \%$ White, $5.2 \%$ Black or African American, and 1.6\% Arabic; gender distribution was $50.6 \%$ female and $49.4 \%$ male; $70.2 \%$ of the school's students were participating in free and reduced meal programs. At the school, all written forms of communication with parents were sent in English and Spanish, thus consent forms in both languages were sent home to parents whose children were in second through fifth grade. For those whose parents provided written consent, children provided verbal assent and took part in the book session to assess their Spanish language abilities in order to take part in the full testing session ( $\sim 10 \mathrm{~min}$ long). As a token of our appreciation for their participation, the school was given a monetary gift card.

\section{Materials and Procedure}

While in their school, children were tested oneon-one with an experimenter in a quiet room apart from their classroom. Due to time constraints, however, children did not complete the Dialect Judgment task and parents were not asked to complete questionnaires. Otherwise, the materials and procedure were identical to those of Study 1. 


\section{Results}

See Figure 4 for results. We conducted a GEE repeated-measures generalized logistic regression using the age groups in the present study (7- to 9- and 10- to 12-year old) and mismatch conditions (race, gender) as factors. The analysis revealed an age group effect (Wald $\chi^{2}=14.81, d f=1, p<.001$ ), in which 10 - to 12-year-olds $(M=0.60, S D=0.49)$ made selections based on dialect significantly more than 7- to 9-year-olds $(M=0.41, S D=0.49)$. We did not find a main effect of mismatch condition nor a Mismatch Condition $\times$ Age Group interaction.

Of particular interest was how performance in Study 4 compared with that of the same age groups (namely 7-9 and 10-12 years) of children from Study 1 . Thus, we conducted a GEE repeated-measures generalized logistic regression using mismatch condition (race, gender), age group (7-9 and 1012 years old), and Study (1 [Suburban], 4 [Urban]) as factors. The analysis revealed that age group predicted performance (Wald $\chi^{2}=17.85, d f=1$, $p<.001)$; post hoc analyses using a Bonferroni correction revealed that 10 - to 12 -year-olds $(M=0.57$, $S D=0.50)$ were more likely than 7- to 9-year-olds $(M=0.41, S D=0.49)$ to predict friendships based on dialect. There were no significant main effects of study or mismatch condition. The analysis also revealed an interaction between mismatch conditions and age groups (Wald $\chi^{2}=4.96, d f=1$, $p=.026$ ); post hoc analyses using a Bonferroni correction revealed that 10 - to 12 -year-olds were more

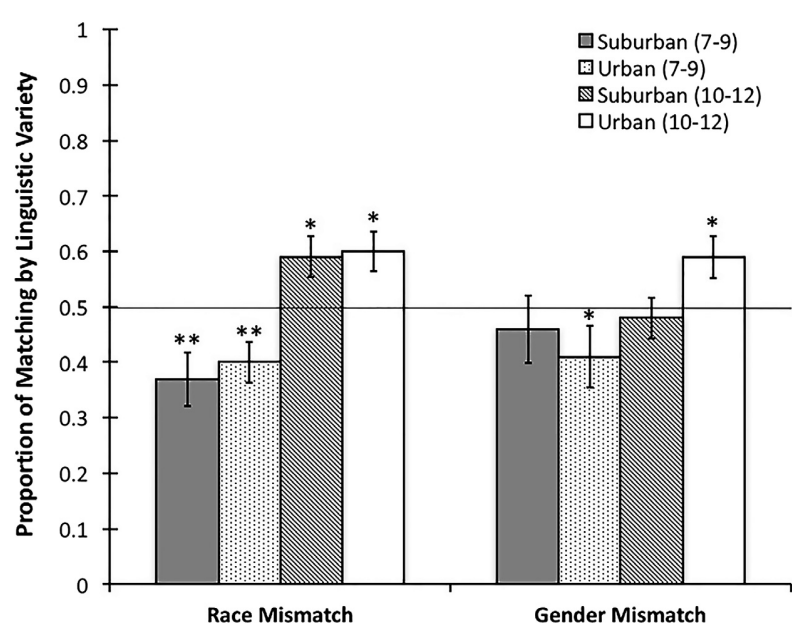

Figure 4. Results from race and gender mismatch conditions for 7- to 9-year and 10- to 12-year olds in Study 1 (suburban sample) and Study 4 (urban sample). Bars depict standard error. ${ }^{*} p<.05$ and $* * p<.01$ indicate the result of a Wilcoxon signed-rank onesample $t$-test to chance (0.50). likely to match by Spanish varieties during race mismatch trials $(M=0.60, S D=0.49)$ than gender mismatch trials $(M=0.54, S D=0.50), p<.001$, whereas 7- to 9-year-olds were more likely to match by Spanish varieties during gender mismatch trials $(M=0.44, S D=0.50)$ than race mismatch trials $(M=0.38, S D=0.49)$. The analysis did not reveal any further significant interactions.

\section{Discussion}

Age group differences in Study 4 replicate those of Study 1, showing that for Spanish-English bilingual children growing up in a U.S. neighborhood in which Hispanics are the dominant population, there is once again a sharp increase between 7-9 and 10-12 years of age in their use of Spanish varieties when making third-person friendship judgments. Surprisingly, these patterns are identical for the urban sample in this study as for the suburban sample of Study 1. Children living in Southwest Detroit "Mexicantown" were living in a neighborhood that is, as seen in the demographics of the school, largely composed of a Hispanic population, and thus were more likely to encounter Spanish speakers and may even have had more experiences with Mexican Spanish than those children in the suburban setting. Nonetheless, we found no significant differences in children's social judgments in these age groups. One explanation is that living in a U.S. context, in which Spanish speakers are treated by the dominant culture as an undifferentiated group, may reduce any sensitivity to the social consequences of Spanish dialects. Perhaps, in their direct experiences, language differences (English vs. Spanish) are more predictive of friendship patterns than are dialects (Mexican vs. Puerto Rican Spanish).

\section{General Discussion}

A key function of social categorization is to support reasoning about how people associate, form social relationships, and belong to one's own versus other social groups (Rhodes, 2012, 2013; Rhodes \& Chalik, 2013). In a U.S. context in which English is the majority language and individuals of Spanish descent are labeled using "pan-ethnic" terms (e.g., Latinos, Hispanic), we investigated when in development Spanish-speaking children associate varieties of their heritage language with social judgments about others. Prior work suggests that monolingual children use language varieties (e.g., foreign 
vs. native accents) as early as infancy when making social preferences (Kinzler, Dupoux, et al., 2012; Kinzler, Shutts, et al., 2012; Kinzler et al., 2007, 2010). Yet little is known regarding third-person social inferences, and studies have shown inconclusive results regarding when in development children use dialect varieties (Kinzler \& DeJesus, 2013). In the present work, we asked Spanish-English bilingual children to complete a Friendship Judgment task that pitted varieties of language (Studies 1, 3, and 4: Mexican Spanish vs. Puerto Rican Spanish; Study 2: English vs. Spanish) against either race or gender. In line with an exemplar-based model of speech perception (Johnson, 2006) that suggests linguistic tokens activate social categories, we hypothesized that children would be sensitive to Spanish dialects early in development when making third-person friendship inferences. Alternatively, given that Spanish is a minority language in the United States, children's sensitivity to Spanish dialects might increase with age. The results revealed that bilingual children make friendship judgments regarding others (i.e., a third-person context) based on languages during early childhood (4-6 years) but based on dialects only later in development (10-12 years). The studies provide important evidence to support the theoretical interpretation that dialects mark social relationships but (at least in the current U.S. context) develop gradually from early childhood through adolescence.

The present research found that older children were more likely than younger children to predict friendships based on Spanish variety; however, this tendency also appears to depend on the competing factor (race or gender). Study 1 found that by 1012 years, children were likely to use Spanish varieties for friendship judgments when pitted against race only. By adolescence (13-17 years), children used Spanish varieties for friendship judgments when pitted against either race or gender. In Study 4 , we replicated this age effect with a sample from a community with a larger Hispanic population and found that 10- to 12 year-old children used Spanish varieties for their friendship judgments when pitted against either race or gender (not race only as in Study 1). Although children might differ in the types of social interactions with others from diverse backgrounds when living in different communities, there were no significant differences between the responses for Studies 1 and 4. However, given the small sample sizes, this lack of differences should be treated with caution.

Interestingly, young bilingual children were no more likely to make use of Spanish dialects in their friendship judgments than were same-aged monolingual speakers of English (Study 3). However, young children's pattern of not using Spanish dialects did not reflect an inability to use language as a cue for third-person friendship inferences, because in Study 2, 4- to 6-year-olds consistently used a speaker's language (English, Spanish). In prior work when race and language were pitted against one another, 5- to 6-year-old monolingual children typically reported that they wanted to be friends (first-person judgments) with those who spoke the same language or with the same accent, regardless of race (Kinzler et al., 2007, 2009). Thus, our findings extend this work in showing that young bilinguals make third-person friendship judgments based on language, regardless of race and gender (Byers-Heinlein et al., 2016; Kinzler, Dupoux, et al., 2012; Kinzler, Shutts, et al., 2012; Souza et al., 2013).

A key puzzling result from the present studies is why children failed to use dialect in their friendship judgments early in development but did so at older ages. Even those children growing up in social contexts where they were more likely to encounter Mexican speakers (Study 4) or whose parents are Mexican (Study 1) were no more successful than other Spanish speakers in making friendship inferences based on Spanish dialects that included Mexican Spanish. Thus, despite immersion in a linguistic environment in which Mexican Spanish was most likely spoken, children's selections were still not based on dialect differences early in development. We suggest three inter related factors that reflect developmental patterns in combination with children's knowledge of their heritage language. First, with age, children become increasingly sensitive to within-language dialect differences (Floccia, Butler, Girard, \& Goslin, 2009; Girard et al., 2008; Wagner, Clopper, \& Pate, 2014). In addition to the Friendship Judgment task, children (in Studies 1, 2, and 3) completed a Dialect Judgment task, in which participants heard sound clips and indicated where a child was from (Mexico or Puerto Rico). The Dialect Judgment task revealed that young bilingual children (4-6 years) were able to distinguish the two Spanish dialects only minimally (above chance in Study 1, but at chance in Study 2) and that the ability to discriminate the two Spanish dialects increased substantially with age. Young children's low level of performance is consistent with prior work showing a marked developmental increase in children's sensitivity to dialect differences in the elementary school years, in which 5- to 6-year-olds distinguished between native and foreign-accented 
speech but did not begin to distinguish regional accented speech until 7 years of age (Floccia et al., 2009; Girard et al., 2008; Wagner et al., 2014). Our task differs in that we examined broadly national variations of Spanish dialects, and not regional dialect or foreign-accented speech, but nonetheless the developmental patterns were quite similar.

Second, becoming aware of language varieties and making social meaning out of dialect differences may reflect children's increasing awareness of, and knowledge about, different regional, cultural, and ethnic groups (e.g., Kinzler \& DeJesus, 2013; Roberts \& Gelman, 2016). With age, children are learning about specific subgroups of Latinos within the United States (e.g., "Mexican," "Puerto Rican"), which may heighten their awareness of the linguistic correlates of these groups. The use of dialects to infer friendships shows that with age, children begin to understand that those who share a dialect may also share a similar culture (Weatherhead et al., 2016) and that individuals who share a culture may prefer those individuals when forming friendships (Rhodes, 2013).

Finally, with age comes a wider range of experiences, including experiences with those who are different from the self. Older children may have more encounters or come into contact with speakers of a wider variety of dialects. Given older children's broader social network, especially in school settings, richer experiences might support the mechanisms guiding older children's social judgments. Future research would be needed to test these ideas more directly.

Together, these three inter related factors suggest that with age, bilingual children's increasing and direct experiences with dialects in their language(s) may support their developmental sensitivity between language varieties to social categories and social relationships. Previous studies with monolingual adults who were asked to distinguish varieties of American English across different U.S. regions have revealed that individuals who directly interacted with a wide-variety of speakers from different U.S. regions were more sensitive in perceiving regional variations, in comparison to those without direct experience (Clopper, Levi, \& Pisoni, 2006; Clopper \& Pisoni, 2004a, 2004b). These studies suggest that, even in adulthood, individuals struggle to distinguish unfamiliar varieties of their native language, but more importantly that linguistic details along with information about a speaker, social groups, and their context are stored by means of direct experiences. Although it is plausible that the present results reflect limited experiences with speakers of their minority language, another possibility is that children are able to detect dialect differences early in development (as revealed by the Dialect Judgment task) but do not find such differences important for social inference (i.e., friendship judgments). Nevertheless, by age 10-12, bilingual Latino children may have overall accumulated a valuable amount of linguistic and cultural experiences to begin using dialect features in their heritage language for making judgments about others without explicit instruction of these dialect differences.

\section{Limitations and Future Directions}

Numbering more than 50 million, Latinos are the largest minority group in the United States and their children now constitute the "majority minority" ethnic group of students in public schools (U.S. Census, 2014). It is, therefore, vital to undertake investigations that aim to understand how Latino children are constructing their social world as they incorporate their two languages (i.e., English and Spanish) and cultures. The present work is limited by the inclusion of participants from a variety of Spanish ethnic backgrounds whose sensitivity to dialect distinctions may vary depending on which dialects they have experienced, as well as their knowledge of associated social, cultural, and ethnic differences. However, we believe this is representative of how diversely rich are Latino children's experiences in the United States. In addition, most participants had at least one parent originally from a country outside the United States and may have experienced accented speech in their parents' use of English. If so, this may have signaled to children that dialect is irrelevant as a social category marker, especially early in development. It would be interesting for future work to examine more systematically whether children whose parents do not speak English with an accent are more likely to use dialect to inform friendship judgments.

Another interesting question for future research is whether children growing up in a monolingual community where Spanish is the majority language would be more sensitive to dialect differences as marking meaningful social differences. For example, children who were born and raised in Mexico may use Spanish varieties to make friendship judgments earlier in development than those born and raised in the United States. However, because racial categories in the United States are different from those across Latin America, in which countries vary in their views of racial category lines and in their 
make-up of White, Indigenous, and Afro-Latino populations, a replication of the present experiments would require adjusting the tasks/materials to be culturally appropriate.

In addition, the Dialect Judgment task used a methodological paradigm that provided participants with an example of what a Mexican and Puerto Rican speaker sound like, which may have taught them the distinction in the context of the task itself. Although labeling the speakers in the task's instructions were included simply to help children understand the task, it is possible that the explicit nature of the task may be measuring developmental capacities of auditory discrimination rather than children's intuitive awareness of dialect variations. An alternative to the task would be to allow participants to freely sort sentences rather than give them a forced choice (see Clopper \& Pisoni, 2007). However, we also carried out a post hoc pilot study that revealed English-speaking undergraduate college students $(n=25,13$ female, 12 male) are on average $62.5 \%, S D=14.6$, above chance, $t(24)=4.37, p<.001$, accurate in distinguishing Spanish dialects during the Dialect Judgment task. Monolingual adults' poor performance suggests that the task's speech exemplars are not sufficient for learning the dialect distinctions. Thus, the present research provides evidence that Spanish-English bilingual children distinguish features of Mexican and Puerto Rican speech prior to using them in third-person judgments.

One value of the present research is that it examined a developmental process in a minority sample that considered the diverse bilingual child's experience (Garcia-Coll et al., 1996). However, for minority bilingual children, the links among language, social inferences, and understanding of ethnicity remain largely unexplored. Relatedly, another important question for future research is how awareness of linguistic varieties in the heritage language may shape bilingual children's friendship formation with in-group members as well as their ethnic identity development. In a recent study, Arredondo, Rosado, and Satterfield (2016) show that children's proficiency in the heritage language supports ethnic identity formation by bolstering the formation of positive relationships and communication among members of their in-group, including both children and their parents (see also Oh \& Fuligni, 2010). Furthermore, being proficient in one's heritage language is associated with identifying closely with the ethnicity of one's family (e.g., Argentinian, Mexican, Puerto Rican) rather than using the American pan-ethnic label to the ethnic group (Fuligni, Kiang, Witkow, \& Baldelomar, 2008; Geerlings et al., 2015; Oh \& Fuligni, 2010; see also Porter, Rheinschmidt-Same, \& Richeson, 2016; Rakic, Steffens, \& Mummendey, 2011). Future research should address whether knowledge of dialects in the heritage language influences children's own social preferences and supports ethnic identity, proficiency in the heritage language, and friendship formation during adolescence.

\section{Conclusion}

As the largest minority group in the United States, Latinos cross multiple linguistic and cultural social categories. The present work demonstrates that early in development, U.S. Spanish-English bilingual children begin to distinguish dialects in their heritage language Spanish (e.g., Mexican vs. Puerto Rican Spanish). However, not until 10 years of age do children begin using such dialects to make social judgments about others. While more work is still necessary to address the root of developmental changes in young bilingual children's sensitivity to dialect variations in their language(s), the present studies encourage future research to consider racial-ethnic and socioeconomic diverse samples to broaden our understanding of cognitive development theoretical concepts as applied to children's real world.

\section{References}

Arredondo, M. M., Rosado, M., \& Satterfield, T. (2016). Understanding the impact of heritage language on ethnic identity formation and literacy for U.S. Latino children. Journal of Cognition and Culture, 16, 245-266. https: / / doi.org/10.1163/15685373-12342179

Byers-Heinlein, K. (2014). Languages as categories: Reframing the "one language or two" question in early bilingual development. Language Learning, 64, 184-201. https: / / doi.org/10.1111/lang.12055

Byers-Heinlein, K., Behrend, D., Said, L. M., Giris, H., \& Poulin-Dubois, D. (2016). Monolingual and bilingual children's social preferences for monolingual and bilingual speakers. Developmental Science. https://doi.org/ 10.1111/desc.12392

Clopper, C. G., Levi, S. V., \& Pisoni, D. B. (2006). Perceptual similarity of regional dialects of American English. Journal of the Acoustical Society of America, 119, 566-574. https: / /doi.org/10.1121/1.2141171

Clopper, C. G., \& Pisoni, D. (2004a). Some acoustic cues for the perceptual categorization of American English regional dialects. Journal of Phonetics, 32, 111-140.

Clopper, C. G., \& Pisoni, D. (2004b). Homebodies and army brats: Some effects of early linguistic experience 
and residential history on dialect categorization. Language Variation and Change, 16, 31-48. https://doi.org/ $10.1017 /$ S0954394504161036

Clopper, C. G., \& Pisoni, D. B. (2007). Free classification of regional dialects of American English. Journal of Phonetics, 35, 421-438.

Cohen, E., \& Haun, D. (2013). The development of tagbased cooperation via a socially acquired trait. Evolution and Human Behavior, 34, 230-235. https://doi.org/ 10.1016/j.evolhumbehav.2013.02.001

Diesendruck, G., Goldfein, R., Rhodes, M., Gelman, S. A., \& Neumark, N. (2013). Cross-cultural differences in children's beliefs about the objectivity of social categories. Child Development, 84, 1906-1917. https://doi. org $/ 10.1111 /$ cdev.12108

Diesendruck, G., \& haLevi, H. (2006). The role of language, appearance, and culture in children's social category-based induction. Child Development, 77, 539-553. https: / / doi.org/10.1111/j.1467-8624.2006.00889.x

Feliciano, C. (2009). Education and ethnic identity formation among children of Latin American and Caribbean immigrants. Sociological Perspectives, 52, 135-158.

Félix-Ortiz, M., Newcomb, M. D., \& Myers, H. (1994). A multidimensional measure of cultural identity for Latino and Latina adolescents. Hispanic Journal of Behavioral Sciences, 16, 99-115.

Floccia, C., Butler, J., Girard, F., \& Goslin, J. (2009). Categorization of regional and foreign accent in 5- to 7year-old British children. International Journal of Behavioral Development, 33, 366-375. https://doi.org/10. $1177 / 0165025409103871$

Fuligni, A. J., Kiang, L., Witkow, M. R., \& Baldelomar, O. (2008). Stability and change in ethnic labeling among adolescents from Asian and Latin American immigrant families. Child Development, 79, 944-956. https://doi. org/10.1111/j.1467-8624.2008.01169.x

García-Coll, G., Crnic, K., Lamberty, G., Wasik, B. H., Jenkins, R., Vázquez García, H., \& McAdoo, H. P. (1996). An integrative model for the study of developmental competencies in minority children. Child Development, 67, 1891-1914. https://doi.org/10.1111/j.14678624.1996.tb01834.x

Garcia-Coll, C., Crnic, K., Lamberty, G., Wasik, B. H., Jenkins, R., . . . McAdoo, H. P. (2008). An integrative model for the study of developmental competencies in minority children. Child Development, 67, 18911914.

Geerlings, J., Verkuyten, M., \& Thijs, J. (2015). Changes in ethnic self-identification and heritage language preference in adolescence: A cross-lagged panel study. Journal of Language and Social Psychology, 34, 501-520. https:/ / doi.org/10.1177/0261927x14564467

Gil-White, F. J. (2001). Are ethnic groups biological "species" to the human brain? Essentialism in our cognition of some social categories. Current Anthropology, 42, 515554. https://doi.org/10.1086/321802

Gimenez, M. E. (1989). "Latino/Hispanic"—who needs a name? The case against standardized terminology.
International Journal of Health Services, 19, 557-571. https: / / doi.org/10.2190/hn6n-p1th-8chl-kw5x

Girard, F., Floccia, C., \& Goslin, J. (2008). Perception and awareness of accents in young children. British Journal of Developmental Psychology, 26, 409-433. https://doi. org/10.1348/026151007X251712

Gutiérrez, D. G. (2004). The Columbia history of Latinos in the United States since 1960. New York, NY: Columbia University Press.

Hirschfeld, L. A. (1996). Race in the making. Cambridge, MA: MIT Press.

Hualde, J. I. (2005). The sounds of Spanish. Cambridge, MA: University Press.

Johnson, K. (2006). Resonance in an exemplar-based lexicon: The emergence of social identity and phonology. Journal of Phonetics, 34, 485-499.

Kinzler, K. D., \& Dautel, J. B. (2012). Children's essentialist reasoning about language and race. Developmental Science, 15, 131-138. https://doi.org/10.1111/j.14677687.2011.01101.x

Kinzler, K. D., \& DeJesus, J. M. (2013). Northern = smart and southern $=$ nice: The development of accent attitudes in the U. S. Quarterly Journal of Experimental Psychology, 66, 1146-1158. https://doi.org/10.1080/ 17470218.2012.731695

Kinzler, K. D., Dupoux, E., \& Spelke, E. S. (2007). The native language of social cognition. Proceedings of the National Academy of Sciences of the United States of America, 104, 12577-12580. https://doi.org/10.1073/pnas. 0705345104

Kinzler, K. D., Dupoux, E., \& Spelke, E. S. (2012). "Native" objects and collaborators: Infants' object choices and acts of giving reflect favor for native over foreign speakers. Journal of Cognition and Development, 13, 67-81. https://doi.org/10.1080/15248372.2011. 567200

Kinzler, K. D., Shutts, K., \& Correll, J. (2010). Priorities in social categories. European Journal of Social Psychology, 40, 581-592. https://doi.org/10.1002/ejsp.739

Kinzler, K. D., Shutts, K., DeJesus, J., \& Spelke, E. S. (2009). Accent trumps race in guiding children's social preferences. Social Cognition, 27, 623-634. https://doi. org/ 10.1521/soco.2009.27.4.623

Kinzler, K. D., Shutts, K., \& Spelke, E. S. (2012). Language-based social preferences among children in South Africa. Language Learning and Development, 8, 215-232. https:/ / doi.org/10.1080/15475441.2011.583611

Labov, W. (2006). A sociolinguistic perspective on sociophonetic research. Journal of Phonetics, 34, 500-515.

Masuoka, N. (2006). Together they become one: examining the predictors of panethnic group consciousness among Asian Americans and Latinos. Social Science Quarterly, 87, 993-1011. https://doi.org/10.1111/j.15406237.2006.00412.x

Nicoladis, E. (1998). First clues to the existence of two input languages: Pragmatic and lexical differentiation in a bilingual child. Bilingualism: Language and Cognition, 1, 105-116. https:/ / doi.org/10.1017/s1366728998000236 
Oboler, N. (1995). Ethnic Labels, Latino Lives : Identity and the Politics of $(R e)$ Presentation in the United States. Minneapolis: University of Minnesota Press.

Oh, J. S., \& Fuligni, A. J. (2010). The role of heritage language development in the ethnic identity and family relationships of adolescents from immigrant backgrounds. Social Development, 19, 202-220.

Petitto, L. A., Katerelos, M., Levy, B. G., Gauna, K., Tétreault, K., \& Ferraro, V. (2001). Bilingual signed and spoken language acquisition from birth: Implications for the mechanisms underlying early bilingual language acquisition. Journal of Child Language, 28, 453-496. https://doi.org/10.1017/s0305000901004718

Porter, S. C., Rheinschmidt-Same, M., \& Richeson, J. A. (2016). Inferring identity from language: Linguistic intergroup bias informs social categorization. Psychological Science, 27, 94-102. https://doi.org/10.1177/ 0956797615612202

Rakic, T., Steffens, M. C., \& Mummendey, A. (2011). Blinded by the accent! The minor role of looks in ethnic categorization. Journal of Personality and Social Psychology, 100, 16-29. https: / /doi.org/10.1037/a0021522

Resnick, M. C. (1980). Phonological variants and dialect identification in Latin American Spanish. Boston, MA: De Gruyter Mouton.

Rhodes, M. (2012). Naïve theories of social groups. Child Development, 83, 1900-1916. https://doi.org/10.1111/j. 1467-8624.2012.01835.x

Rhodes, M. (2013). How two intuitive theories shape the development of social categorization. Child Development Perspectives, 7, 12-16. https://doi.org/10.1111/cdep.12007

Rhodes, M., \& Chalik, L. (2013). Social categories as markers of intrinsic interpersonal obligations. Psychological Science, 24, 999-1006. https://doi.org/10.1177/ 0956797612466267

Rhodes, M., \& Gelman, S. A. (2009). A developmental examination of the conceptual structure of animal, artifact, and human social categories across two cultural contexts. Cognitive Psychology, 59, 244-274. https://doi. org/10.1016/j.cogpsych.2009.05.001

Roberts, S. O., \& Gelman, S. A. (2015). Do children see in black and white? Children's and adults' categorizations of multiracial individuals. Child Development, 86, 18301847. https://doi.org/10.1111/cdev.12410

Roberts, S. O., \& Gelman, S. A. (2016). Can White children grow up to be Black? Children's reasoning about the stability of emotion and race. Developmental Psychology, 52, 887-893. https://doi.org/10.1037/dev0000132

Roberts, S. O., Williams, A. D., \& Gelman, S. A. (2016). Children's and adults' predictions of black, white, and multiracial friendship patterns. Journal of Cognition and Development. https:/ /doi.org/10.1080/15248372.2016.1262374

Scarry, R. (2011). Richard Scarry's best busy year ever. New York, NY: Sterling Publishing.

Souza, A. L., Byers-Heinlein, K., \& Poulin-Dubois, D. (2013). Bilingual and monolingual children prefer native-accented speakers. Frontiers in Psychology, 4(953), 1-6. https: / / doi.org/10.3389/fpsyg.2013.00953
Tare, M., \& Gelman, S. A. (2010). Can you say it another way? Cognitive factors in bilingual children's pragmatic language skills. Journal of Cognition and Development, 11, 137-158. https://doi.org/ 10.1080/ 15248371003699951

Taylor, M. G. (1996). The development of children's beliefs about social and biological aspects of gender differences. Child Development, 67, 1555-1571. https://doi. org/10.2307/1131718

Taylor, P., Lopez, M. H., Martínez, J. H., \& Velasco, G. (2012). When labels don't fit: Hispanics and their views of identity. Washington, DC: Pew Hispanic Center.

Taylor, M. G., Rhodes, M., \& Gelman, S. A. (2009). Boys will be boys; cows will be cows: Children's essentialist reasoning about gender categories and animal species. Child Development, 80, 461-481. https://doi.org/10. $1111 / \mathrm{j} .1467-8624.2009 .01272 . x$

U.S. Census Bureau. (2014). Profile America facts for feaures: CB15-FF.18 Hispanic heritage month 2015. Retrieved from: https://www.census.gov/content/dam/Census/ newsroom/facts-for-features/2015/cb15-ff18_hispanic_ heritage_month.pdf

U.S. Census Bureau. (2016). QuickFacts: Ann Arbor city, Michigan. Retrieved from: https://www.census.gov/ quickfacts / fact/table/annarborcitymichigan/PST045216

Valdés, G. (2001). Heritage language students: Profiles and possibilities. In J. K. Peyton \& S. McGinnis (Eds.), Heritage languages in America: Blueprint for the future (pp. 37-77). Washington, DC and McHenry, IL: Center for Applied Linguistics and Delta Systems.

Van Bezooijen, R., \& Gooskens, C. (1999). Identification of language varieties: The contribution of different linguistic levels. Journal of Language and Social Psychology, 18, 31-48. https://doi.org/10.1177/0261927x9901800 1003

Wagner, L., Clopper, C. G., \& Pate, J. K. (2014). Children's perception of dialect variation. Journal of Child Language, 41, 1062-1084. https://doi.org/10.1017/ S0305000913000330

Waxman, S. R. (2012). Social categories are shaped by social experience. Trends in Cognitive Sciences, 16, 531532. https://doi.org/10.1016/j.tics.2012.09.007

Weatherhead, D., White, K. S., \& Friedman, O. (2016). Where are you from? Preschoolers infer background from accent. Journal of Experimental Child Psychology, 143, 171-178. https://doi.org/10.1016/j.jecp.2015.10.011

\section{Supporting Information}

Additional supporting information may be found in the online version of this article at the publisher's website:

Appendix S1. Methodological Details of the Friendships Judgment Task

Appendix S2. Additional Analysis for Study 1 